\title{
PAX7 is required for patterning the esophageal musculature
}

\author{
Daisuke Chihara', Anthony I. Romer ${ }^{1,2,5}$, C. Florian Bentzinger ${ }^{3,4,6}$, Michael A. Rudnicki ${ }^{3,4}$ and Robert S. Krauss ${ }^{1,2^{*}}$
}

\begin{abstract}
Background: The mammalian esophageal musculature is unique in that it makes a transition from smooth to skeletal muscle, with most of this process occurring after birth. In order to better understand the mechanisms that control esophageal musculature development, we investigated the roles in this process of the paired box transcription factor, PAX7, a principal regulator of skeletal myogenic progenitor cells. Previous studies showed that Pax7 is important for determining the esophageal muscle composition.

Results: We characterized the postnatal development of the esophageal musculature in $\mathrm{Pax}^{-1}$ mice by analyzing morphology, muscle composition, and the expression of markers of myogenesis, cell proliferation, and apoptosis. $\mathrm{Pax}^{-1-}$ mice displayed megaesophagus with a severe defect in the postnatal developmental process whereby esophageal smooth muscle is replaced by skeletal muscle. $\mathrm{Pax}^{-1-}$ esophagi have substantially reduced skeletal muscle, most likely due to diminished proliferation and premature differentiation of skeletal muscle precursor cells. This impaired the proximal-to-distal progression of skeletal myogenesis and indirectly affected the patterning of the smooth muscle-containing portion of the esophageal musculature.

Conclusions: Postnatal patterning of the esophageal musculature appears to require robust, PAX7-dependent cell proliferation to drive the proximal-to-distal progression of skeletal myogenesis. This process in turn influences distal smooth muscle morphogenesis and development of the mature pattern of the esophageal musculature.
\end{abstract}

Keywords: Esophageal myogenesis, Megaesophagus, Pax7, Skeletal muscle, Smooth muscle, Cell proliferation, Frontal expansion

\section{Background}

The musculature of the esophagus controls passage of food into the stomach by waves of peristaltic contractions. The lower esophageal sphincter (LES) is a bundle of smooth muscles at the distal end of the esophagus, where it meets the stomach. During swallowing, tonic smooth muscles of the LES relax briefly in order to allow passage of food into the stomach [1]. Esophageal muscles are adversely affected in a number of human disorders, including myotonic dystrophy, oculopharyngeal muscular dystrophy, and the inflammatory myopathies; this may lead to dysphagia, regurgitation, choking while eating, and other symptoms. Disorders of LES

\footnotetext{
*Correspondence: Robert.Krauss@mssm.edu

${ }^{1}$ Department of Developmental and Regenerative Biology, Mount Sinai School of Medicine, New York, NY 10029, USA

${ }^{2}$ Graduate School of Biological Sciences, One Gustave L. Levy Place, Icahn

School of Medicine at Mount Sinai, New York, NY 10029, USA

Full list of author information is available at the end of the article
}

function include gastroesophageal reflux disease (GERD) and achalasia, the latter characterized by impaired relaxation of the LES and perturbed peristalsis, often resulting in megaesophagus. The primary cause of achalasia is defective signaling between the NO-producing inhibitory myenteric neurons and neighboring smooth muscle cells (SMCs) of the LES $[2,3]$. Although studies have identified several mutant mouse lines that display these esophageal defects, their etiologies are poorly understood.

The mammalian esophageal musculature is unique in that it makes a transition from smooth to skeletal muscle, with most of this process occurring after birth. The esophagus is ensheathed by the muscularis externa (ME) $[1,4,5]$. Skeletal muscle comprises the proximal portion of the ME and is critical for swallowing and proximal waves of peristalsis. Smooth muscle surrounds the distal portion, including the esophagogastric junction, which harbors the LES. During mammalian development, the ME initially comprises only smooth muscle. 
In the mouse, skeletal muscle precursors are first detected in the proximal ME at embryonic day (E) 13 [6]. Over the next 3 weeks of life, smooth muscle is replaced by skeletal muscle in a proximal-to-distal manner, and the adult ME pattern is nearly completed by postnatal day (P) 14 [7-9]. Human esophageal myogenesis is similar, although smooth muscle is maintained in a more proximal position than in the mouse, but is still restricted to the distal one-third of the esophagus [10]. Interestingly, the amount and distribution of skeletal muscle varies among individuals $[11,12]$. The developmental and cell biological mechanisms that underlie the smooth to skeletal muscle replacement process have been controversial, with multiple mechanisms proposed [7-9, 13-15] (reviewed in ref. [13]). Rishniw et al. [9] were the first to suggest that some sort of distal compaction of smooth muscle cells was important in the replacement of smooth muscle with skeletal muscle. However, the mechanisms for this process remained unclear.

Our previous study provided insight into mechanisms that control morphogenesis of the esophageal ME [13]. We showed that mice lacking the multifunctional cell surface receptor Cdo (also called Cdon) have a defect in the postnatal developmental process whereby esophageal smooth muscle is replaced by skeletal muscle. Analyses of various skeletal myogenic markers revealed that proliferative skeletal muscle progenitor cells migrated in a transition zone (TZ) along the length of the esophagus in a proximal-to-distal manner, leaving differentiated myofibers in its wake. Distal to the TZ, smooth muscle fascicles underwent a morphogenetic process whereby they changed their orientation relative to each other and to the lumen. In a distal-to-proximal manner, smooth muscle fascicles altered their orientation from parallel to the lumen to nearly perpendicular to the lumen. Upon completion of the process, smooth muscle occupied only the most distal part of the esophagus and the LES. $\mathrm{Cdo}^{-/-}$mice had no defect in skeletal myogenesis or in the numbers of various skeletal muscle progenitor cell types in the TZ. Rather, they were specifically defective in SMC fascicular reorientation, leading to ectopic, proximal fascicles displaying an inappropriate orientation and an aberrantly proximal skeletalsmooth muscle boundary [13]. Importantly, $\mathrm{Cdo}^{-/-}$mice also displayed megaesophagus and achalasia, with a smooth muscle cell-intrinsic defect in NO-mediated LES relaxation. Taken together, our previous findings identified a mechanism of ME maturation and provided a link between esophageal morphogenesis and motility disorders. Although the ME maturation defect in $C d o^{-/-}$mice is almost certainly smooth muscle cell-autonomous, a role for TZ-based skeletal myogenesis in promoting the fascicular reorientation process seems likely.

Multiple mechanisms have also been proposed for the developmental origin of esophageal skeletal muscles
(ESMs). Minchin et al. reported that, similar to trunk and limb muscles, ESMs arise from Pax3/Pax7-expressing cells that originate in somites [16]. However, Gopalakrishnan et al. have recently demonstrated that ESMs are derived from the cardiopharyngeal mesoderm, and their development is dependent on expression of $T b x 1$ and Isl1, genes important for specific muscles of the head but not the trunk [17]. These factors lie upstream of the myogenic bHLH factors, MyoD and myogenin, which promote commitment and differentiation of cells in the skeletal muscle lineage [18]. Consistent with a cranial mesoderm origin for ESMs, Pax3 was not expressed during development of these muscles and was dispensable for their development in mice [17].

In contrast to Pax3, Pax7 is expressed in skeletal muscle precursors of the esophageal TZ [13, 19-22]. Importantly, however, Pax7 is never expressed in the SMC lineage as shown by lineage tracing studies, immunochemistry, and in situ hybridization [13, 19-22]. Worl et al. used electron microscopy to show that $\operatorname{Pax} 7^{-1}$ mice had a reduced number of skeletal muscle precursor cells in the esophageal ME, defective esophageal skeletal myogenesis, and an aberrantly proximal skeletal-smooth muscle boundary [14]. As Pax7 is not expressed in the smooth muscle lineage, such mice offer an opportunity to ask whether TZ-based skeletal myogenesis might affect smooth muscle patterning in the esophagus. We report here that $\operatorname{Pax}^{\prime-}$ mice have a severe defect in the postnatal developmental process whereby esophageal smooth muscle is replaced by skeletal muscle. Our results suggest that this defect is due to reduced cell proliferation in the $\mathrm{TZ}$, probably due to precocious differentiation of skeletal muscle precursor cells, leading to a deficit in skeletal muscle. This defect in $\operatorname{Pax} 7^{-1-}$ mice is associated with both megaesophagus and defective maturation of esophageal smooth muscle patterning. Our findings add to the previous model for esophageal musculature development. PAX7-dependent cell proliferation in the $\mathrm{TZ}$ is important for the proximal-to-distal progression of esophageal skeletal myogenesis. Furthermore, loss of PAX7 leads to a non-autonomous defect that influences smooth muscle fascicle reorientation and development of a mature ME.

\section{Methods}

Mice

$\mathrm{Pax}^{+/-}$mice were originally obtained from Peter Gruss [20] and are from the colony maintained by the Rudnicki laboratory. These mice are on a $129 \mathrm{~Sv}$ background. It has previously been reported that on a C57BL/6J background, $\mathrm{Pax} 7^{+/-}$esophagi have a mild defect in development of the esophageal musculature [14]. On the 129Sv background, we have not observed differences in esophageal development between $\mathrm{Pax} 7^{+/+}$and $\mathrm{Pax} 7^{+/}$mice, and 
at P21 they were indistinguishable in esophageal length, location of the skeletal-smooth muscle boundary, and luminal diameter (Additional file 1: Figure S1). Therefore, we used both $\operatorname{Pax}^{T^{++}}$and $\operatorname{Pax} 7^{+/}$animals as controls. All animal procedures were conducted in accordance with institutional guidelines for the care and use of laboratory animals as approved by the Institutional Animal Care and Use Committees (IACUC) of the Icahn School of Medicine at Mount Sinai and according to the Canadian Council on Animal Care guidelines and the University of Ottawa Animal Care Committee protocols.

\section{Preparation of frozen sections}

Dissected esophagi were prepared for histology by directly freezing in OCT. For longitudinal sections, incisions were made along the entire esophagus in order to clear out the ingesta, and the sheet of dissected esophagus was laid flat prior to freezing. Frozen tissue blocks were sectioned at $10 \mu \mathrm{m}$ and placed on Superfrost Plus slides (Thermo Fisher Scientific).

\section{Immunofluorescence and histology}

Frozen sections $(10 \mu \mathrm{m})$ were immunostained as previously described [13]. Slides were fixed in $4 \%$ PFA, washed in PBS, permeabilized in $0.3 \%$ Triton/PBS, washed in PBS, blocked in $10 \%$ goat serum, and incubated overnight at $4{ }^{\circ} \mathrm{C}$ with primary antibodies in blocking buffer. Additional M.O.M. blocking (Vector Laboratories) steps were performed according to the manufacturer's instructions when mouse primary antibodies were used. Antibodies used included mouse anti-sarcomeric actin (5C5; 1:1000; Sigma-Aldrich), rabbit anti- $\alpha$-smooth muscle actin (1:200; Abcam), rabbit anti-MyoD (1:500; Santa Cruz Biotechnology, Inc.), mouse anti-myogenin (F5D; 1:50; Santa Cruz Biotechnology, Inc.), mouse anti-Mcadherin (1:75; Santa Cruz Biotechnology, Inc.), rabbit antinNOS (C12H1; 1:500; Cell Signaling Technology), rabbit anti-Ki67 (1:1000; Leica), mouse anti-Ki67 (1:100; BD Pharmingen), and rabbit anti-phospho-Histone H3 (ser10) (1:1000; Millipore). After permeabilization, the following antibodies required an antigen retrieval step of boiling the sections in $10 \mathrm{mM}$ sodium citrate: mouse anti-Pax7 (1:100; Developmental Studies Hybridoma Bank, Iowa City, IA), and rabbit cleaved caspase-3 (Asp175; 1:1000; Cell Signaling Technology). Fluorescent secondary antibodies were from Invitrogen and used at 1:1000: goat antirabbit Alexa Fluor 488, anti-rabbit Alexa Fluor 568, goat anti-mouse Alexa Fluor 488, and goat anti-mouse IgG1 Alexa Fluor 647. Nuclei were fluorescently labeled with DAPI and mounted with Fluoromount-G anti-fade medium (SouthernBiotech). TUNEL assay was performed using the In Situ Cell Death Detection kit from Roche. For Figs. 4 and 5 and Additional file 2: S2, the transition zone was identified by adjacent longitudinal sections stained with antibodies to $\langle$-smooth muscle actin and sarcomeric actin.

Hematoxylin and eosin (H\&E) staining was performed as described [23]. Sections were dehydrated through graded ethanol and xylene and mounted with Permount (Fisher Scientific).

\section{Microscopy}

Microscopy was performed at the Mount Sinai Microscopy Shared Resource Facility using Axioplan2 and Axioplan2 IE microscopes (Carl Zeiss) equipped with $\times 10, \times 20, \times 40$, and $\times 100$ objective lenses that had numerical apertures of 0.5 , $0.8,0.75$, and 1.4, respectively. Images were acquired at room temperature with a camera (Axio Cam MRm; Carl Zeiss). Mosaic images were compiled using the stitching feature in the AxioVision software. The maximum luminal diameter and length of esophageal skeletal/smooth muscle were measured by using ImageJ software (National Institutes of Health).

\section{Statistical analysis}

Data were expressed as mean \pm standard deviation. Differences were tested by two-tailed $t$ test. Values with $P<0.05$ were considered statistically significant.

\section{Results}

\section{$\mathrm{Pax}^{-/-}$mice develop megaesophagus}

Most $\operatorname{Pax}^{\prime-}$ mice die within 3 weeks of birth [20]. Surviving P21 $\mathrm{Pax}^{-1-}$ mice displayed megaesophagus with $100 \%$ penetrance. P21 $\mathrm{Pax}^{-/}$esophagi had an enlarged diameter relative to $\mathrm{Pax}^{+/+}$and $\mathrm{Pax}^{+/-}$esophagi, and a dilated lumen filled with partially digested food (Fig. 1a-d). No other gross abnormalities were observed in the GI tracts of $\mathrm{Pax} \mathrm{T}^{--}$mice. P21 $\mathrm{Pax} 7^{+/}$esophagi resembled the wild type in esophageal length, location of the skeletal-smooth muscle boundary, and luminal diameter (Additional file 1: Figure S1); therefore, $\operatorname{Pax} 7^{1 /+}$ and $\mathrm{Pax}^{+/-}$esophagi were used interchangeably as controls. Immunofluorescence analysis (IFA) of longitudinal sections of P21 esophagi with markers of smooth muscle $(\alpha$-smooth muscle actin ( $\alpha S M A))$ and skeletal muscle (sarcomeric actin (SA)) revealed that $\operatorname{Pax}^{-1}$ mice have a mispatterned ME, with the skeletal-smooth muscle boundary occurring at an abnormally proximal position (Fig. 1c, d). Most smooth muscle in the esophagi of Pax $7^{+/-}$mice was found in a short, broad segment at the esophagogastric junction, where SMCs were bundled into fascicles that were stacked side by side with an orientation perpendicular to the lumen (Fig. 1i). In contrast, distal to the aberrantly proximal boundary in $\mathrm{Pax}^{-1-}$ esophagi, there was a long, thin, ectopic extension of smooth muscle that connected to the LES (Fig. 1d, j). The fascicles in this ectopic region of smooth muscle in $\mathrm{Pax}^{--}$esophagi were parallel to the 

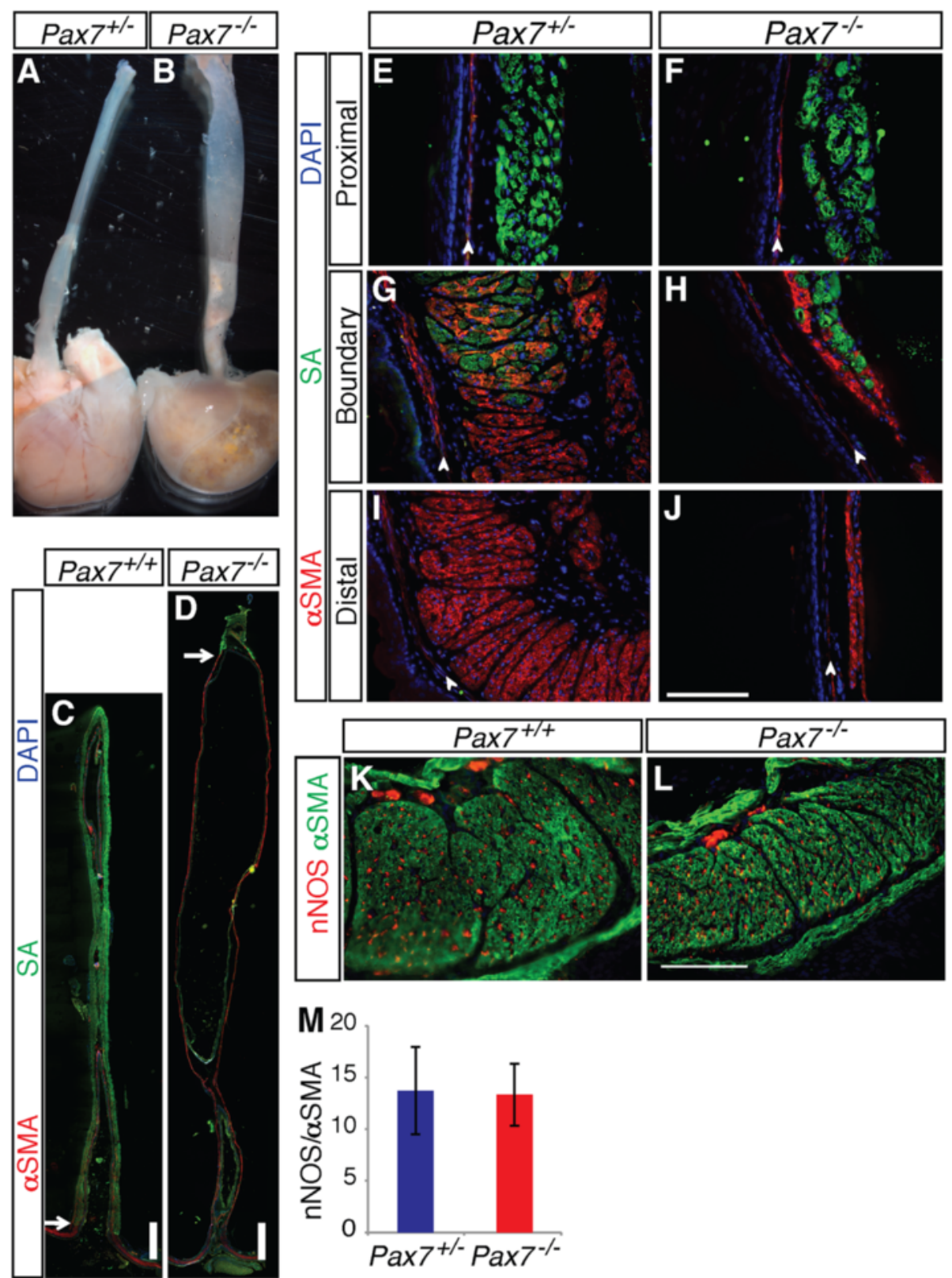

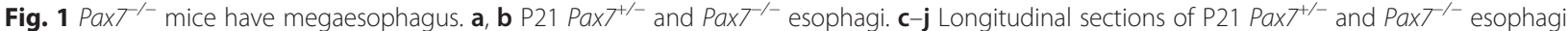
were stained with antibodies to a-smooth muscle actin (aSMA; red) and sarcomeric actin (SA; green) for smooth and skeletal muscles, respectively. c, $\mathbf{d} \mathrm{Pax}^{-/-}$esophagi have an aberrantly proximally located skeletal-smooth muscle boundary (arrows). Note that the Pax $7^{+/+}$esophagus is cut off at the top end, making it appear shorter than the Pax $7^{-1-}$ esophagus, the full length of which is shown. In fact, at P21, Pax $7^{-1-}$ esophagi are shorter than $\mathrm{Pax} T^{+/+}$esophagi (see Fig. 3e). e, $\mathbf{f}$ The proximal ME of $\mathrm{PaxT^{-- }}$ esophagi has skeletal muscle of relatively normal appearance. $\mathbf{g}, \mathbf{h}$ Smooth muscle cells are dispersed with skeletal myofibers around the skeletal-smooth muscle boundary (Boundary). Note that the ME of the Pax $7^{-1-}$ esophagus is thinner than the control at the skeletal-smooth muscle boundary. $\mathbf{i}, \mathbf{j}$ The distal Pax $7^{-1-}$ esophagus has a long, thin extension of smooth muscle instead of the short, broad segment of smooth muscle found in controls. $\mathbf{e}-\mathbf{j}$ The thin aSMA ${ }^{+}$layer adjacent to the ME is the muscularis mucosa (arrowheads). $\mathbf{k}$ I Longitudinal sections through the LES of P21 Pax $7^{1 /+}$ and Pax $7^{-1}$ esophagi mice were stained with antibodies to aSMA (green) and nNOS (red) to label inhibitory intramural neurons. $\mathbf{m}$ Quantification of the relative numbers of nNOS ${ }^{+}$cells to aSMA staining in the ME of the LES. Values are means $\pm S D, n=5$. Bars: (c, d) $1 \mathrm{~mm} ;(\mathbf{e}-\mathbf{l}) 0.2 \mathrm{~mm}$

lumen, indicating they had failed to undergo the normal reorientation process. A few SMCs were dispersed within the skeletal muscle at the skeletal-smooth muscle boundary in both $\mathrm{Pax}^{+/-}$and $\mathrm{Pax}^{--}$esophagi (Fig. 1g, h).
Despite the mispatterned and thinner ME distal to the aberrantly proximal boundary in $\mathrm{Pax} 7^{-1}$ esophagi, the morphology of the smooth muscle fascicles appeared normal in the $\operatorname{Pax}^{-1}$ LES (Fig. 1k, l). The Pax $7^{-1}$ LES was 
somewhat thinner than that of the control mice (control $=135.8 \pm 9.6 \mathrm{~mm}(n=5)$; mutant $=92.0 \pm 12.9 \mathrm{~mm}$ $(n=6) ; p<0.01)$. This may be due to a combination of defective development of the ME and the overall growth retardation displayed by these mice [20]. The ME of the proximal portion of $\mathrm{Pax} 7^{-1}$ esophagi was similar to that of $\mathrm{Pax}^{+/-}$esophagi, although the skeletal myofibers in mutants appeared to have a greater amount of between-fiber space than in controls (Fig. 1f).

Megaesophagus is sometimes associated with loss of nitrergic neurons in the myenteric plexus of the LES [3]. We therefore quantified the number of neurons in the LES from P21 $\mathrm{Pax}^{1 /+}$ and $\mathrm{Pax} 7^{-1}$ mice. IFA of LES sections with an antibody to nNOS revealed that the number and pattern of nitrergic neurons were similar between control and mutant mice (Fig. 1k-m). Taken together, P21 $\mathrm{Pax}^{-1}$ mice showed megaesophagus with an aberrantly proximal skeletal-smooth muscle boundary. The morphological defects of the P21 $\mathrm{Pax}^{/-} \mathrm{ME}$ were most apparent in the region between the skeletalsmooth muscle boundary and LES, but were not as severe in the proximal region and in the LES itself.

\section{Defective ME patterning in $\mathrm{Pax}^{-/-}$esophagi}

To determine when these defects in $\operatorname{Pax} 7^{/-}$mice arose, cross sections were taken from P0-P21 at proximal, midlevel, and distal regions. Expression of $\alpha \mathrm{SMA}$ and SA was assessed. The proximal and distal ME were composed of skeletal and smooth muscles, respectively, and the cross sections of proximal and distal $\mathrm{Pax}^{/-}$esophagi showed no obvious defects at P0, P7, and P21 (Fig. 2a-f, m-r, s, u). Mid-level cross sections revealed that esophageal dilation became recognizable in $\mathrm{Pax} 7^{-1}$ esophagi at P7 (Fig. $2 \mathrm{~g}-\mathrm{j}, \mathrm{t}$ ), and this distention was more severe at P21 (Fig. 2k, l, t). H\&E-stained cross sections revealed that the P21 Pax7 $7^{-1-}$ ME was thinner than that of controls in the mid-level region (Fig. $2 \mathrm{v}-\mathrm{x}$ ). In contrast to the control mid-level ME, which was composed of skeletal muscle at P7 and P21, the $\mathrm{Pax}^{-1-}$ mid-level ME displayed only smooth muscle at these time points (Fig. 2i-l).

These findings are consistent with the aberrant skeletal-smooth muscle boundary seen in P21 $\mathrm{Pax}^{/-}$ esophagi (Fig. 1d), and they suggested a defect in the process whereby smooth muscle is replaced by skeletal muscle in these animals. We therefore analyzed the proximal-to-distal progression of skeletal myogenesis during ME development. Expression of aSMA and SA was assessed in longitudinal sections taken at P0, P7, and P21 (Figs. 1c, d and 3a-d). The distal-most skeletal muscle cells in these sections were embedded in smooth muscle. Progression of ME development was monitored by measuring the distance from the distal-most $\mathrm{SA}^{+}$cell to the LES. At P0, this distance was about half of the length in $\mathrm{Pax}^{+/-}$esophagi, whereas it constituted nearly $80 \%$ of the total length in Pax $^{/-}$esophagi (Fig. 3a, b, e). The distance from the distal-most $\mathrm{SA}^{+}$cell to the LES diminished in $\mathrm{Pax}^{+/-}$esophagi from P0 to P21, and the distal-most $\mathrm{SA}^{+}$cell was found just proximal to the LES at P21 (Figs. 1c and 3a, c, e). In contrast, the distal-most SA ${ }^{+}$ cell in $\operatorname{Pax}^{-1}$ animals was at a significantly more proximal location than in $\mathrm{Pax}^{+/}$animals as early as PO and maintained through P21 (Figs. 1d and 3b, d, e). The distance from the distal-most $\mathrm{SA}^{+}$cell to the LES slightly decreased in $\mathrm{Pax}^{-1}$ esophagi by P21; however, the distance still constituted $\sim 65 \%$ of the total length (Fig. 3e). The total length of $\mathrm{Pax}^{-1}$ esophagi is $\sim 20 \%$ shorter than that of control animals by P21 (Fig. 3e) [14]. This is likely due to overall growth retardation in these mice [20], but cannot alone account for the ME patterning defects.

\section{$\mathrm{Pax}^{-1-}$ esophagi have reduced number of cells undergoing myogenic differentiation}

To analyze the Pax7 expression pattern in developing esophageal ME, longitudinal sections of $\mathrm{Pax}^{+/}$esophagi were taken at P7 and P21 and stained with an antibody for Pax7. At P7, most Pax7 $7^{+}$cells were found in the $\mathrm{TZ}$, and their number decreased at more proximal locations (Fig. 4a, c, e, g). Pax $7^{+}$cells diminished from the entire esophagi at P21, when proximal-to-distal progression of skeletal muscle was complete (Fig. 4b, d, f, g). At this stage, relatively few $\operatorname{Pax} 7^{+}$cells were present, as these probably mainly represent the population of satellite cells seen in the adult esophagus [13,24].

We hypothesized that impaired proximal-to-distal progression of skeletal myogenesis in $\mathrm{Pax}^{-1}$ esophagi resulted from a decrease in the number of cells undergoing myogenic differentiation. We therefore assessed the expression of MyoD (a marker of determined myoblasts) and myogenin (a marker of differentiating myoblasts) during ME development. Longitudinal sections of P7 $\mathrm{Pax} 7^{+-}$ and $\mathrm{Pax}^{-1}$ esophagi were stained with antibodies for MyoD and myogenin. Consistent with our previous study [13], most $\mathrm{MyoD}^{+}$and myogenin ${ }^{+}$cells were found in the $\mathrm{TZ}$ and their numbers diminished at more proximal locations in control animals. Pax $7^{1-}$ mice had $\sim 30 \%$ the number of $\mathrm{MyoD}^{+}$cells and $\sim 50 \%$ the number of myogenin $^{+}$cells as control mice (Fig. $5 \mathrm{a}-\mathrm{f}$ ).

Reduction of the number of cells expressing these markers of myogenesis may result from a decrease in the muscle progenitor cell population, either by alteration in cell proliferation or cell survival. To investigate cell proliferation in the developing ME, longitudinal sections were taken from P7 $\mathrm{Pax}^{+/-}$and $\mathrm{Pax} \mathrm{T}^{1-}$ esophagi and stained with antibodies for Ki67 and phospho-histone H3. Most $\mathrm{Ki}^{6} 7^{+}$and phospho-histone $\mathrm{H}^{+}$cells were found in the TZ in PaxT $^{+/-}$esophagi (Fig. $5 \mathrm{~g}, \mathrm{k}, \mathrm{l}, \mathrm{p}$ ). Much lower numbers of $\mathrm{Ki}^{+} 7^{+}$and phospho-histone $\mathrm{H}^{+}$ 

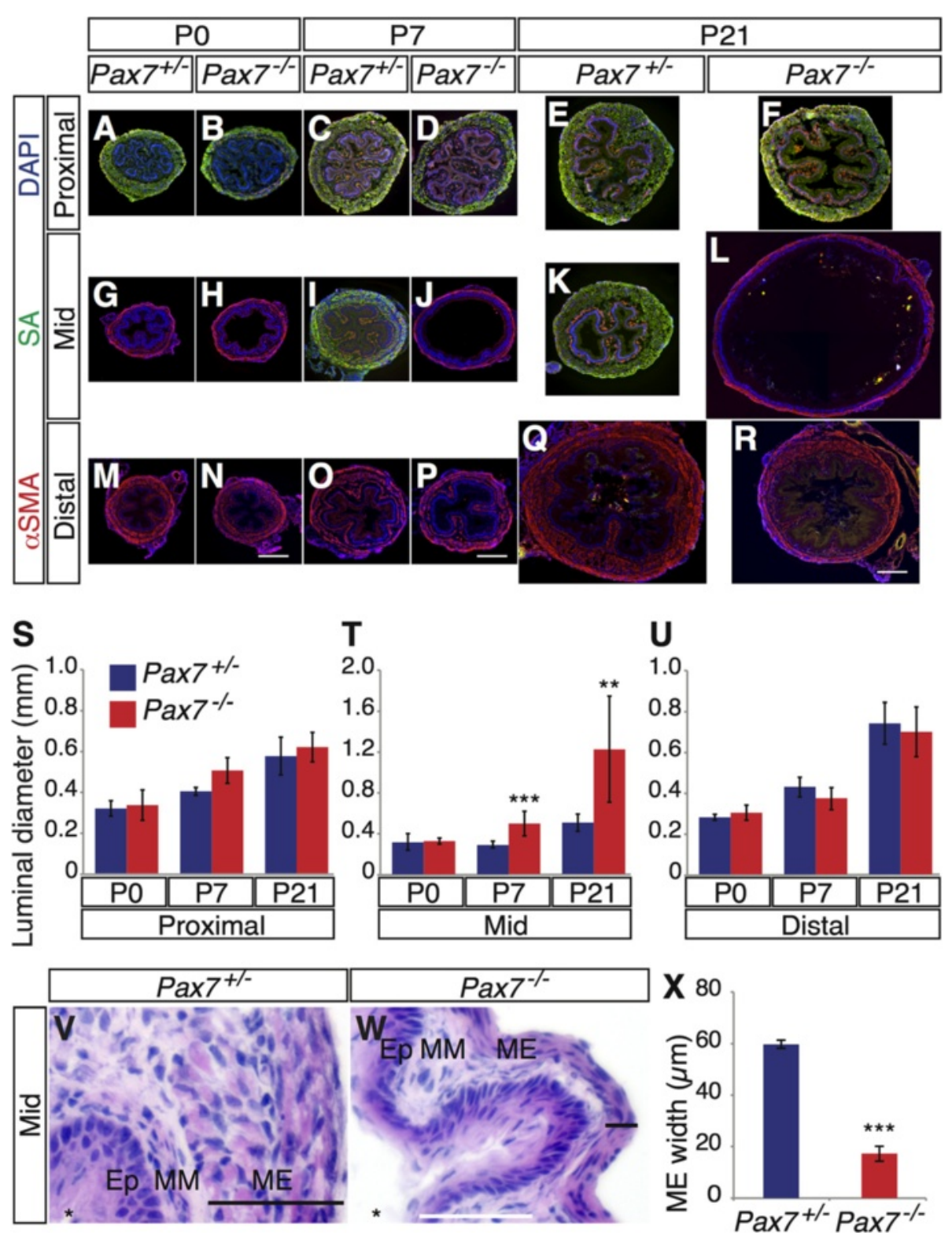

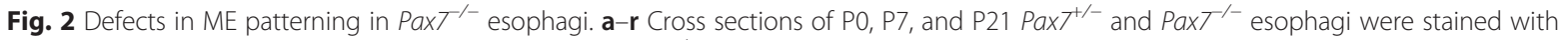
antibodies to aSMA, SA, and DAPI. j, I The P7 and P21 Pax $7^{-/-}$esophagi are dilated, and skeletal muscle is replaced by smooth muscle at the

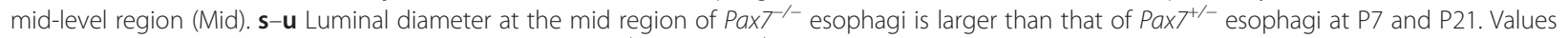

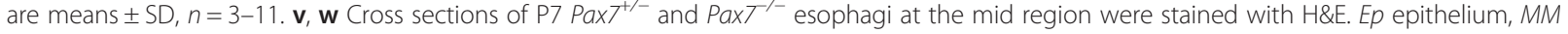
muscularis mucosa, ME muscularis externa. The black horizontal bars indicate the width of the ME; the asterisks indicate the lumens. $\mathbf{x}$ Quantification of the width of the ME, as shown in ( $\mathbf{v}$ and $\mathbf{w})$. Values are means $\pm \mathrm{SD}, n=3$. Bars: $(\mathbf{a}-\mathbf{r}) 0.2 \mathrm{~mm},(\mathbf{v}, \mathbf{w}) 50 \mu \mathrm{m}$. ${ }^{* *} P<0.01$; ${ }^{* * *} P<0.001$

cells were found in the Pax $7^{1-}$ TZ (Fig. 5h, k, m, p). Consistent with the largely postmitotic nature of ME smooth muscle cells [13], fewer $\mathrm{Ki}^{+} 7^{+}$and phospho-histone $\mathrm{H}^{+}$ cells were present in the distal ME below the TZ in both Pax $7^{+/-}$and $\mathrm{Pax}^{-/-}$esophagi (Fig. 5i, j, k, n, o, p). Therefore, cell proliferation in the $\mathrm{TZ}$ is substantially reduced in Pax $7^{-1-}$ mice.

To confirm that cell proliferation was reduced in skeletal muscle precursor cells per se, we stained longitudinal
P7 sections with antibodies for MyoD or another lineage marker, M-cadherin, plus Ki67. Pax $^{\prime-}$ mice had $20 \%$ the number of $\mathrm{MyoD}^{+} / \mathrm{Ki}^{+} 7^{+}$cells in the $\mathrm{TZ}$ as control mice (Fig. 5q-w). Pax $7^{/-}$mice had only $\sim 13 \%$ the number of M-cadherin ${ }^{+}$cells as controls (Fig. $5 \mathrm{x}-\mathrm{dd}$ ). Furthermore, $45 \%$ of control M-cadherin ${ }^{+}$cells were also positive for Ki67, whereas none of the small number of $\mathrm{M}$-cadherin ${ }^{+}$cells in the $\mathrm{Pax}^{-1} \mathrm{TZ}$ were also positive for Ki67. These results indicate that cell proliferation of 


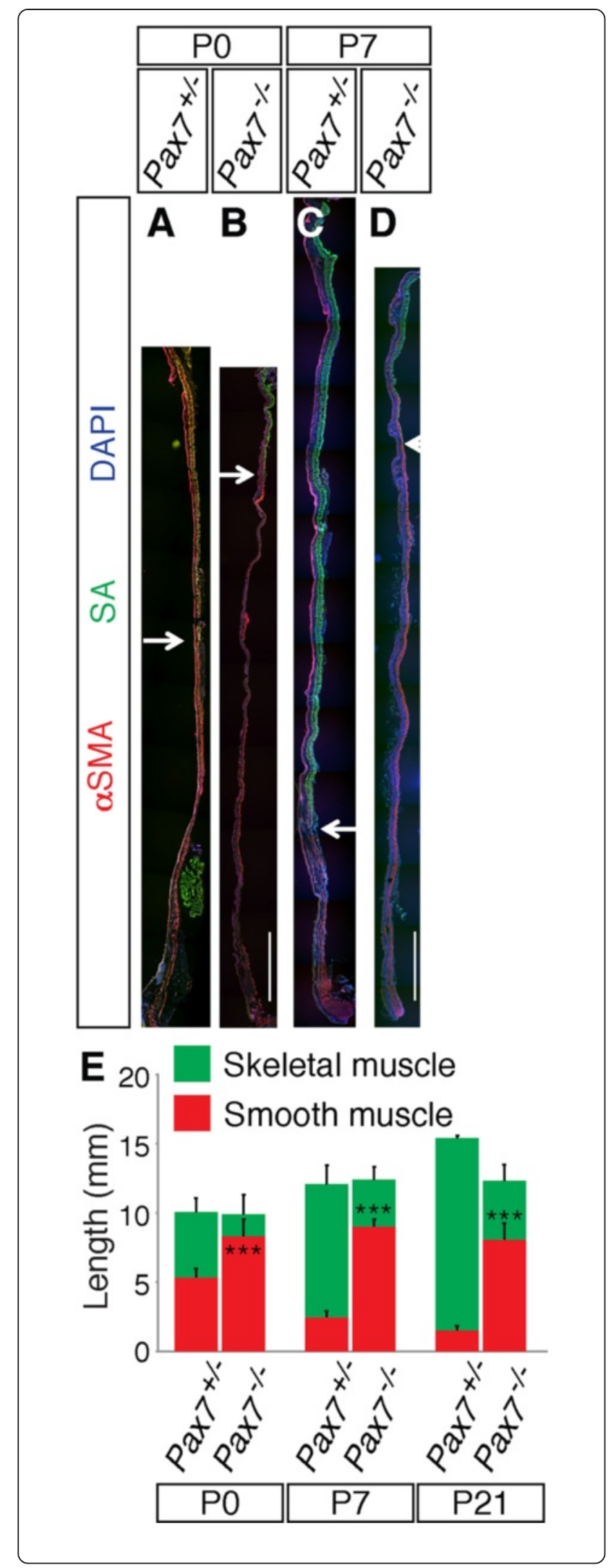

Fig. $3 \mathrm{Pax}^{-1-}$ mice display defective proximal-to-distal progression of ME development. a-d Longitudinal sections of PO and P7 Pax $7^{+-}$ and $\mathrm{Pax}^{-/-}$esophagi were stained with antibodies to aSMA, SA, and DAPI. Pax $T^{1-}$ esophagi have an aberrantly proximally located skeletal-smooth muscle boundary (arrows). The $\mathrm{SA}^{+}$tissue near the distal end of the $\mathrm{Pax}^{+/-}$esophagus in (a) is the diaphragm. e The distance between the distal-most SA ${ }^{+}$cell and the LES was measured and is represented by the red portion of the histogram bars. The distance decreases progressively with age in $\mathrm{Pax}^{7^{+-}}$esophagi, but this fails to occur in $\mathrm{Pax}^{--}$esophagi. Values are means $\pm \mathrm{SD}, n=3-5$. Bars: $1 \mathrm{~mm} .{ }^{* * *} P<0.001$

skeletal muscle precursors is strongly diminished in the Pax $7^{-1}$ esophagus.

We next analyzed longitudinal sections of $\mathrm{P} 7 \mathrm{Pax}^{+/}$ and $\operatorname{Pax}^{-1}$ esophagi by TUNEL assay and by IFA with an antibody to cleaved caspase-3, with thymuses as positive controls. TUNEL ${ }^{+}$and cleaved caspase $-3^{+}$cells were easily detected in control thymuses (Additional file 2: Figure S2E, J). Consistent with previous studies [7-9, 13], no apoptotic cells were observed in $\mathrm{Pax}^{+/}$esophagi (Additional file 2: Figure S2A, C, F, H). Furthermore, no $\mathrm{TUNEL}^{+}$or cleaved caspase- $3^{+}$cells were observed in the esophagi of $\operatorname{Pax}^{--}$mice (Additional file 2: Figure S2B, D, G, I), indicating that the diminished skeletal myogenesis seen in these mice is not a consequence of cell death.

\section{Discussion}

In this study, we investigated the role of $\operatorname{Pax} 7$ in esophageal musculature development. $\mathrm{Pax} 7^{-1}$ mice displayed megaesophagus with a severe defect in the postnatal developmental processes whereby esophageal smooth muscle is replaced by skeletal muscle and the ME acquires a mature pattern. The proximal-to-distal progression of skeletal myogenesis was impaired in $\operatorname{Pax} 7^{-}$ esophagi, associated with a significant reduction in cell proliferation of skeletal muscle precursors in the TZ. Additionally, our findings revealed that Pax7 is important for proper postnatal patterning of the entire ME, including the distal smooth muscle layers.

We previously proposed a model for postnatal development of the esophageal ME [13]. During esophageal ME maturation, skeletal muscle progenitors commit to the myogenic lineage and differentiate within a TZ that migrates along the length of the esophagus in a proximal-to-distal manner. Distal to the TZ, SMCs are largely non-proliferative and bundled into long, thin fascicles. SMC fascicles are initially arranged end-to-end and parallel to the lumen. During ME maturation, SMC fascicles rearrange their orientation such that they are ultimately arranged side by side and nearly perpendicular to the lumen; as a consequence, at the end of $\mathrm{ME}$ maturation, the fascicles occupy only the distal portion of the ME. Fascicular reorientation occurs in a distal-toproximal manner. We suggested that this process "clears 

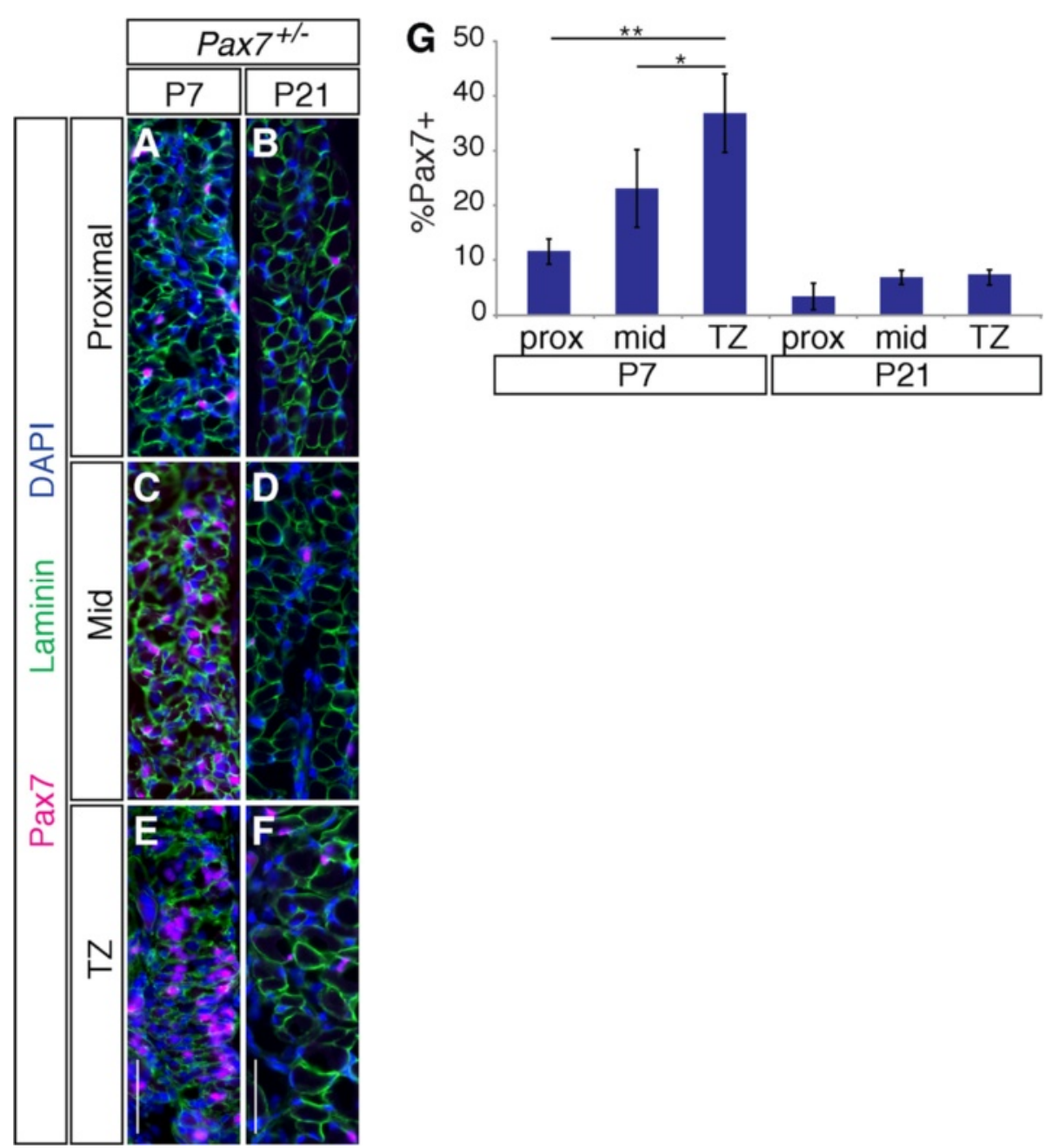

Fig. 4 Expression of Pax7 in the esophageal musculature at P7 and P21. a-f Longitudinal sections of P7 and P21 Pax ${ }^{+/-}$esophagi were stained with antibodies to Pax7, laminin, and DAPI. Proximal, mid-level (Mid), and TZ regions were analyzed. The greatest number of Pax $7^{+}$cells was observed in the TZ at P7, and the numbers diminished in mid and proximal regions. The number of Pax $7^{+}$cells is diminished at P21 when proximal-to-distal progression of ME development is complete. $\mathbf{g}$ Quantification of Pax $7^{+}$cells in proximal (prox), mid, and TZ regions measured as a percentage of total DAPI ${ }^{+}$cells in the ME. Bars: $0.2 \mathrm{~mm}$

a path" for the TZ, which migrates distally and produces the skeletal myofibers that constitute the majority of the length of the mature ME of the adult mouse [13].

Mice lacking the multifunctional cell surface receptor, Cdo, have a mispatterned ME, including an aberrantly proximal skeletal-smooth muscle boundary, and they develop megaesophagus. These mice are specifically defective in SMC fascicular reorientation, without obvious alterations in TZ-based skeletal myogenesis [13]. $\mathrm{Cdo}^{-/-}$ mice had an ectopic, proximal region of smooth muscle in which the fascicles remained in the end-to-end, parallel-to-the-lumen orientation that normally characterizes an earlier developmental stage. This phenotype is very likely to be SMC-autonomous [13]. In contrast, the esophageal defects in $\mathrm{Pax}^{-/}$mice are almost certainly autonomous to the skeletal muscle lineage. $\operatorname{Pax} 7$ is expressed in skeletal muscle precursor cells throughout the body, including the esophageal TZ, but it is never expressed in the SMC lineage [13, 19-22]. Examination of esophageal ME patterning in $\mathrm{Pax}^{-1}$ esophagi revealed that their musculature consisted mainly of smooth muscle, having skeletal muscle only at the very proximal end. This phenotype was more severe in $\operatorname{Pax} 7^{/-}$ esophagi than $\mathrm{Cdo}^{---}$esophagi. Smooth muscle occupied the distal $\sim 20 \%$ of P14 $\mathrm{Cdo}^{-1-}$ esophagi, whereas it occupied the distal $\sim 65 \%$ of P21 Pax $7^{/-}$esophagi. There was no difference in the position of the skeletal-smooth muscle boundary between control and $\mathrm{Cdo}^{-/-}$mice at P0, but $\mathrm{Pax}^{\prime-}$ esophagi already had an aberrantly proximal skeletal-smooth muscle boundary at P0. Moreover, in contrast to $\mathrm{Cdo}^{-/-}$mice, the numbers of $\mathrm{MyoD}^{+}$and myogenin $^{+}$cells were reduced, as was cell proliferation, in the 

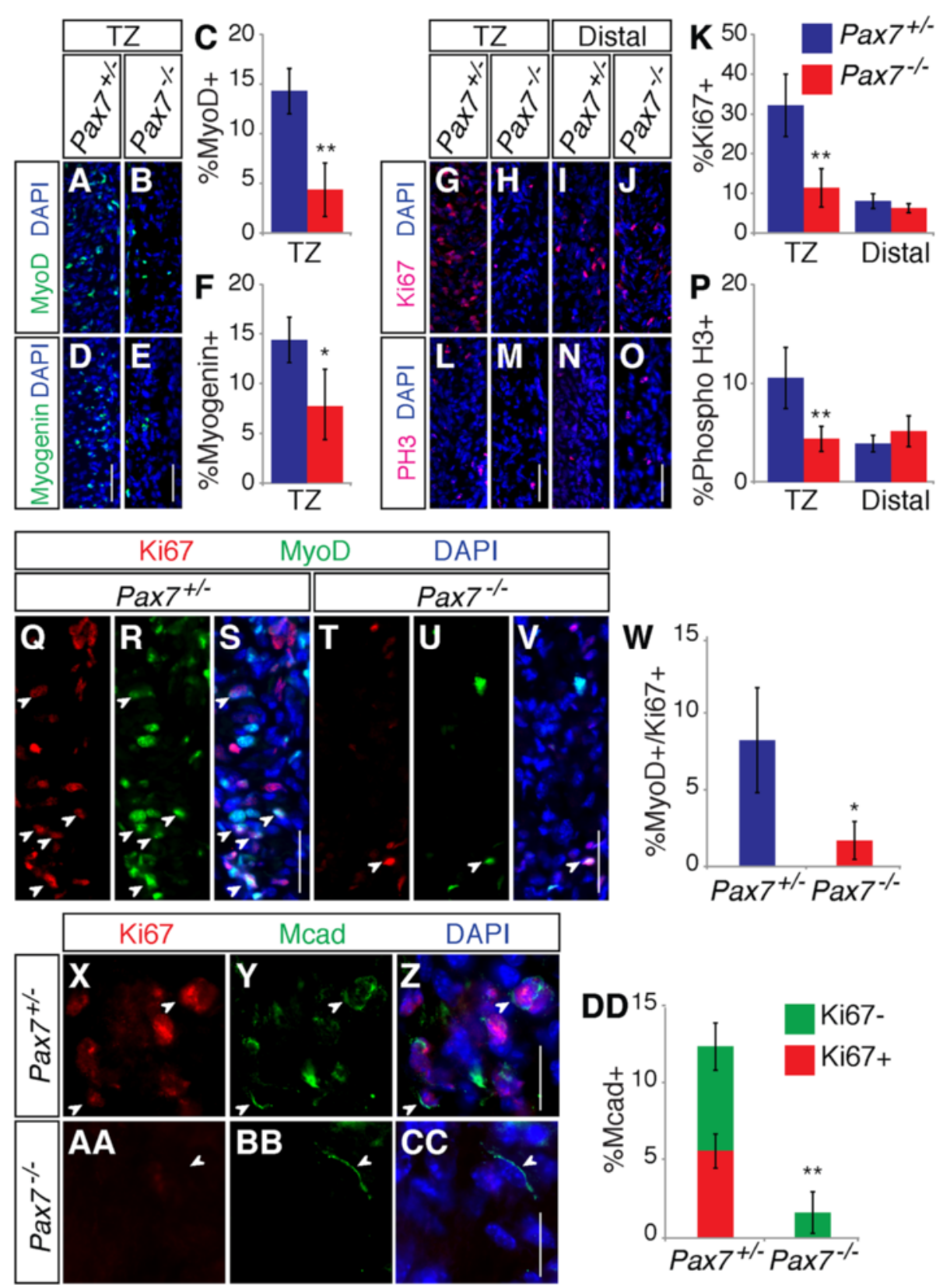

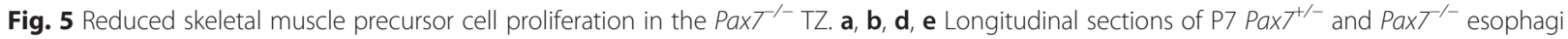
were stained with either MyoD $(\mathbf{a}, \mathbf{b})$ or Myogenin $(\mathbf{d}, \mathbf{e})$ antibodies and DAPI. The TZ was analyzed. $\mathbf{c}, \mathbf{f}$ Quantification of MyoD ${ }^{+}(\mathbf{c})$ and Myogenin ${ }^{+}$ (d) cells within the TZ, measured as a percentage of total DAPI ${ }^{+}$cells in the ME. Values are means \pm SD, $n=3-5$. $\mathbf{g}-\mathbf{j}, \mathbf{l}-\mathbf{o}$ Longitudinal sections of P7

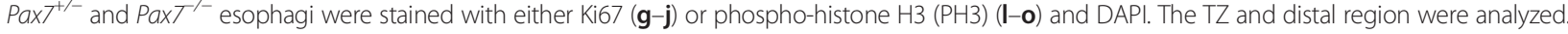
$\mathbf{k}, \mathbf{p}$ Quantification of $\mathrm{Ki}_{6} 7^{+}(\mathbf{k})$ and $\mathrm{PH}^{+}$(p) cells within the $\mathrm{TZ}$ and distal region, measured as a percentage of total DAPI ${ }^{+}$cells in the given region. The numbers of $\mathrm{Ki}_{6} 7^{+}$and $\mathrm{PH}^{+}$cells were reduced in the $\mathrm{TZ}$ in Pax $7^{-1-}$ mice, but not in the distal region. Values are means $\pm \mathrm{SD}, n=4-5$. q-v Longitudinal sections of P7 Pax ${ }^{+/-}$and Pax $7^{-1-}$ esophagi were stained with MyoD and Ki67 antibodies and with DAPI. w Quantification of $\mathrm{MyoD}^{+} / \mathrm{Ki} \mathrm{7}^{+}$cells within the TZ, measured as a percentage of total DAPI ${ }^{+}$cells in the TZ. Values are means $\pm \mathrm{SD}, n=4$. $\mathbf{x}$-cc Longitudinal sections of

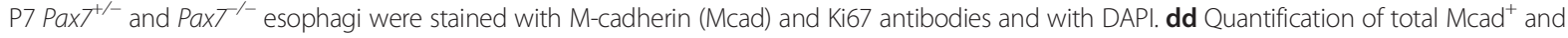
$\mathrm{Mcad}^{+} / \mathrm{Ki}_{\mathrm{G}} \mathrm{7}^{+}$cells within the TZ, measured as a percentage of total DAPI ${ }^{+}$cells in the TZ. Values are means $\pm \mathrm{SD}, n=4$. Bars: $0.2 \mathrm{~mm}(\mathbf{a}-\mathbf{v}) ; 20 \mu \mathrm{m}$ $(\mathbf{x}-\mathbf{c c}) .{ }^{*} P<0.05 ;{ }^{* *} P<0.01$

Pax $7^{-1}$ TZ. Therefore, the aberrantly proximal skeletalsmooth muscle boundary in $\mathrm{Pax}^{-1}$ esophagi resulted from reduced production of ESM.
Distal to the aberrantly proximal skeletal-smooth muscle boundary, the $\operatorname{Pax}^{-1}$ smooth muscle layer was very thin, with the fascicles parallel to the lumen for 
nearly the entire length of the ME. The LES was normally patterned, consistent with it being in the region in which fascicles reorient earliest [13]. As Pax7 is never expressed in the SMC lineage, the mispatterning of ESM in $\operatorname{Pax} 7^{1-}$ esophagi is presumably a non-cell-autonomous effect. It seems likely that smooth muscle mispatterning occurs as a consequence of impaired proximal-to-distal progression of the $\mathrm{Pax}^{-1} \mathrm{TZ}$, but further work will be required to prove this. One physiological consequence of this combination of deficient skeletal myogenesis and SMC fascicle mispatterning is that $\mathrm{Pax}^{--}$mice have an enlarged luminal diameter specifically in the mid region of the esophagus (i.e., megaesophagus). We speculate that smooth muscles, in this abnormal location and fascicular arrangement, are not successful in maintaining the full integrity and function of the ME. This may occur for a variety of non-mutually exclusive reasons, including defective peristalsis, causing the slow passage of food and physical distension; suboptimal innervation; or some combination of these and additional processes.

Our findings support and add to the previous model for esophageal musculature development. Based on the fact that $\operatorname{Pax}^{-1-}$ mice display a presumably non-SMCautonomous defect in fascicular reorientation, we propose the following hypothesis. Smooth muscle fascicular reorientation may be facilitated by forces exerted via the proximal-to-distal progression of the TZ. This progression may "push" the SMCs to a more distal region, where they are eventually exposed to the signals that trigger the rearrangement of SMCs relative to one another, resulting in reorientation of fascicles. Proliferation of skeletal muscle precursor cells was significantly reduced in the $\mathrm{Pax}^{-1-} \mathrm{TZ}$ during esophageal muscular development. This suggests that robust cell proliferation, which presumably participates in driving the proximalto-distal progression of the TZ, depends on PAX7. This is consistent with the known roles of PAX7 in maintaining proliferation and preventing precocious differentiation [24, 25]. Therefore, it is most likely that skeletal muscle precursor cells differentiate prematurely during esophageal myogenesis in the $\mathrm{Pax}^{-/-} \mathrm{TZ}$, resulting in reduced production of ESM.

That robust cell proliferation in the TZ may be critical for the proximal-to-distal progression of skeletal myogenesis is supported by a mathematical model of cell migration [26]. This model predicts that proliferation at the invading front is the key mechanism driving directed migration. These predictions were experimentally validated with the directional migration and colonization of the gut by vagal neural crest cells that establish the enteric nervous system [26]. This "frontal expansion" model, which emphasizes the importance of proliferation to a carrying capacity, is potentially relevant to a wider range of invasion systems such as angiogenesis, epidermal wound healing, and malignant invasion [26]. Therefore, this model may also apply to the proximal-to-distal progression of esophageal skeletal myogenesis.

Megaesophagus is observed as a feature of several animal models of Duchenne muscular dystrophy, including some strains of $m d x$ mice, and some dystrophic dog and cat models [27-30]. It was proposed that megaesophagus in some of these animal models was caused by a hypertrophic diaphragm and ensuing esophageal constriction, a very different scenario than that seen in $\operatorname{Pax}^{-1}$ mice. There is, however, little in the literature to suggest that megaesophagus is common in patients with muscular dystrophy or other myopathies. Megaesophagus occurs more frequently in severe cases of achalasia, as a consequence of inability to fully relax the LES $[2,3]$. It will be of interest for future studies to use electrophysiology and manometry to test if $\operatorname{Pax}^{-1}$ mice have a nonautonomous defect in LES function.

\section{Conclusions}

Our analyses on $\mathrm{Pax}^{-1-}$ esophageal musculature development suggest that ME patterning requires robust, PAX7-dependent cell proliferation in the TZ, which helps drive the proximal-to-distal progression of skeletal myogenesis. This process may in turn be necessary for distal smooth muscle fascicle reorientation and development of a mature ME.

\section{Additional files}

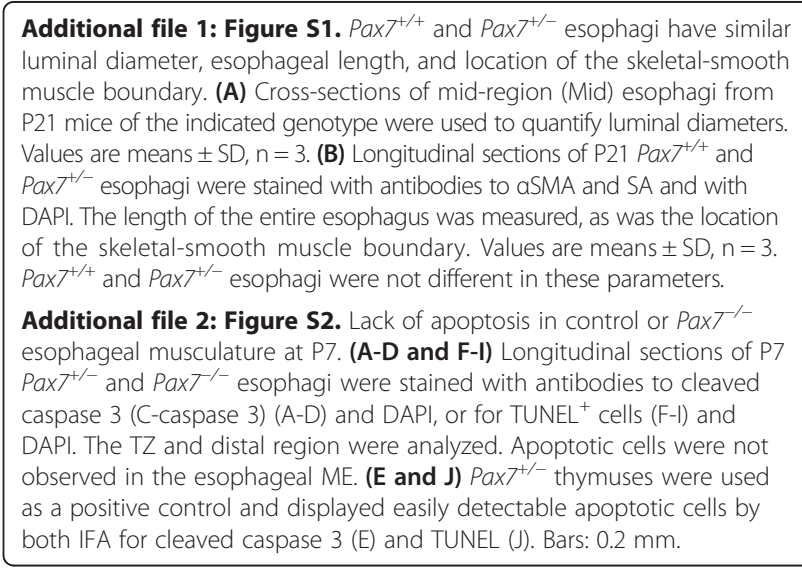

\section{Abbreviations}

CDO: cell adhesion molecule-related/down-regulated by oncogenes; ESM: esophageal skeletal muscle; GERD: gastroesophageal reflux disease; IFA: immunofluorescence analysis; LES: lower esophageal sphincter; ME: muscularis externa; Myf5: myogenic factor 5; MyoD: myoblast determination protein; nNOS: neuronal nitric oxide synthases; NO: nitric oxide; PAX: paired box protein; SA: sarcomeric actin; SMA: smooth muscle actin; SMC: smooth muscle cell; TZ: transition zone.

\section{Competing interests}

The authors declare that they have no competing interests. 


\section{Authors' contributions}

$D C, A R$, and RSK designed the experiments and analyzed the data. DC, AR, and CFB carried out the experiments. CFB and MAR generated and provided critical reagents. DC, AR, CFB, MAR, and RSK were involved in drafting the manuscript and/or revising it critically for important intellectual content. All authors read and approved the final manuscript.

\section{Acknowledgements}

This work was supported by a grant from the NIH (AR46207) to RSK; grants from the NIH (R01AR044031) and Canadian Institutes for Health Research (MOP-12080 and MOP-81288) to MAR. DC was an American Association of Anatomists Scholar, and this research was funded in part by the American Association of Anatomists. CFB was supported by the Swiss National Science Foundation.

\section{Author details}

'Department of Developmental and Regenerative Biology, Mount Sinai School of Medicine, New York, NY 10029, USA. ${ }^{2}$ Graduate School of Biological Sciences, One Gustave L. Levy Place, Icahn School of Medicine at Mount Sinai, New York, NY 10029, USA. ${ }^{3}$ Regenerative Medicine Program, Ottawa Hospital Research Institute, 501 Smyth Road, Ottawa K1H 8 L6 ON, Canada. ${ }^{4}$ Department of Cellular and Molecular Medicine, University of Ottawa, 451 Smyth Road, Ottawa K1H 8 M5 ON, Canada. ${ }^{5}$ Present address: Department of Genetics and Development, Columbia University, 701 West 168th Street, HHSC 1602, New York, NY 10032, USA. ${ }^{6}$ Present address: Nestlé Institute of Health Sciences, EPFL Campus, 1015 Lausanne, Switzerland.

Received: 23 June 2015 Accepted: 7 November 2015

Published online: 03 December 2015

\section{References}

1. Goyal RK, Chaudhury A. Physiology of normal esophageal motility. J Clin Gastroenterol. 2008;42(5):610-9.

2. Goyal RK, Chaudhury A. Pathogenesis of achalasia: lessons from mutant mice. Gastroenterology. 2010;139(4):1086-90

3. Park W, Vaezi MF. Etiology and pathogenesis of achalasia: the current understanding. Am J Gastroenterol. 2005;100(6):1404-14.

4. Mittal RK. Longitudinal muscle of the esophagus: its role in esophageal health and disease. Curr Opin Gastroenterol. 2013;29(4):421-30.

5. Mittal RK, Balaban DH. The esophagogastric junction. N Engl J Med. 1997:336(13):924-32

6. Zhao W, Dhoot GK. Skeletal muscle precursors in mouse esophagus are determined during early fetal development. Dev Dyn. 2000;219(1):10-20.

7. Kablar B, Tajbakhsh S, Rudnicki MA. Transdifferentiation of esophageal smooth to skeletal muscle is myogenic bHLH factor-dependent Development. 2000;127(8):1627-39.

8. Patapoutian A, Wold BJ, Wagner RA. Evidence for developmentally programmed transdifferentiation in mouse esophageal muscle. Science. 1995:270(5243):1818-21.

9. Rishniw M, Fisher PW, Doran RM, Meadows E, Klein WH, Kotlikoff MI. Smooth muscle persists in the muscularis externa of developing and adult mouse esophagus. J Muscle Res Cell Motil. 2007;28(2-3):153-65.

10. Katori Y, Cho BH, Song CH, Fujimiya M, Murakami G, Kawase T. Smooth-tostriated muscle transition in human esophagus: an immunohistochemical study using fetal and adult materials. Ann Anat. 2010;192(1):33-41.

11. Kallmunzer B, Sorensen B, Neuhuber WL, Worl J. Enteric co-innervation of striated muscle fibres in human oesophagus. Neurogastroenterol Motil. 2008;20(6):597-610.

12. Meyer GW, Austin RM, Brady 3rd CE, Castell DO. Muscle anatomy of the human esophagus. J Clin Gastroenterol. 1986:8(2):131-4.

13. Romer Al, Singh J, Rattan S, Krauss RS. Smooth muscle fascicular reorientation is required for esophageal morphogenesis and dependent on Cdo. J Cell Biol. 2013;201(2):309-23.

14. Worl J, Breuer C, Neuhuber WL. Deletion of Pax7 changes the tunica muscularis of the mouse esophagus from an entirely striated into a mixed phenotype. Dev Dyn. 2009;238(4):864-74.

15. Worl J, Neuhuber WL. Ultrastructural analysis of the smooth-to-striated transition zone in the developing mouse esophagus: emphasis on apoptosis of smooth and origin and differentiation of striated muscle cells. Dev Dyn. 2005;233(3):964-82.
16. Minchin JEN, Williams VC, Hinits $Y$, Low S, Tandon P, Fan C-M, et al. Oesophageal and sternohyal muscle fibres are novel Pax3-dependent migratory somite derivatives essential for ingestion. Development. 2013;140(14):2972-84.

17. Gopalakrishnan S, Comai G, Sambasivan R, Francou A, Kelly R, Tajbakhsh S. A cranial mesoderm origin for esophagus striated muscles. Dev Cell. 2015;34(6):694-704.

18. Parker MH, Seale P, Rudnicki MA. Looking back to the embryo: defining transcriptional networks in adult myogenesis. Nat Rev Genet. 2003;4:497-507.

19. Jostes B, Walther C, Gruss P. The murine paired box gene, Pax7, is expressed specifically during the development of the nervous and muscular system. Mech Dev. 1990;33(1):27-37.

20. Mansouri A, Stoykova A, Torres M, Gruss P. Dysgenesis of cephalic neural crest derivatives in Pax7-/- mutant mice. Development. 1996;122(3):831-8.

21. Murdoch B, DelConte C, Garcia-Castro MI. Pax7 lineage contributions to the mammalian neural crest. PLoS One. 2012;7(7):e41089.

22. Seale P, Sabourin LA, Girgis-Gabardo A, Mansouri A, Gruss P, Rudnicki MA. Pax7 is required for the specification of myogenic satellite cells. Cell. 2000;102(6):777-86.

23. Zhang W, Hong M, Bae G-U, Kang J-S, Krauss RS. Boc modifies the holoprosencephaly spectrum of Cdo mutant mice. Dis Model Mech. 2011:4:368-80

24. Zammit PS, Relaix F, Nagata Y, Ruiz AP, Collins CA, Partridge TA, et al. Pax7 and myogenic progression in skeletal muscle satellite cells. J Cell Sci. 2006;119(Pt 9):1824-32

25. von Maltzahn J, Jones AE, Parks RJ, Rudnicki MA. Pax7 is critical for the normal function of satellite cells in adult skeletal muscle. Proc Natl Acad Sci U S A. 2013;110(41):16474-9.

26. Simpson MJ, Zhang DC, Mariani M, Landman KA, Newgreen DF. Cell proliferation drives neural crest cell invasion of the intestine. Dev Biol. 2007:302(2):553-68.

27. Bedu A-S, Labruyère JJ, Thibaud JL, Barthélémy I, Leperlier D, Saunders JH, et al. Age-related thoracic radiographic changes in golden and labrador retriever muscular dystrophy. Vet Radiol Ultrasound. 2012;53(5):492-500.

28. Carpenter J, Hoffman E, Romanul F, Kunkel L, Rosales R, Ma N, et al. Feline muscular dystrophy with dystrophin deficiency. Am J Pathol. 1989;135:909-19.

29. Judge LM, Arnett ALH, Banks GB, Chamberlain JS. Expression of the dystrophin isoform Dp116 preserves functional muscle mass and extends lifespan without preventing dystrophy in severely dystrophic mice. Hum Mol Genet. 2011;20(24):4978-90.

30. Wertz K, Füchtbauer EM. Dmd(mdx-ßgeo): a new allele for the mouse dystrophin gene. Dev Dyn. 1998;212:229-41.

\section{Submit your next manuscript to BioMed Central and take full advantage of:}

- Convenient online submission

- Thorough peer review

- No space constraints or color figure charges

- Immediate publication on acceptance

- Inclusion in PubMed, CAS, Scopus and Google Scholar

- Research which is freely available for redistribution 